\title{
Combination diagnosis with elastography strain ratio and molecular markers effectively improves the diagnosis rate of small breast cancer and lymph node metastasis
}

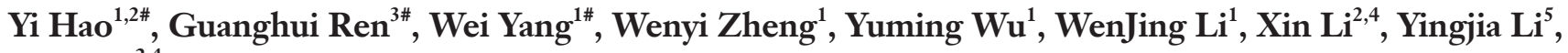 \\ Xia Guo,
}

${ }^{1}$ Department of Ultrasound, ${ }^{2}$ Shenzhen Key Laboratory of Viral Oncology, ${ }^{3}$ Department of General Surgery, ${ }^{4}$ Center for Clinical Research and Innovation (CCRI), Shenzhen Hospital, Southern Medical University, Shenzhen 518100, China; ${ }^{5}$ Department of Ultrasound, Nanfang Hospital, Southern Medical University, Guangzhou 510515, China

"These authors contributed equally to this work.

Correspondence to: Xia Guo, PhD. Shenzhen Key Laboratory of Viral Oncology, Center for Clinical Research and Innovation (CCRI), Shenzhen Hospital, Southern Medical University, No. 1333 Xinhu Road, Shenzhen 518100, China. Email: guoxia0504_sz@163.com; Yingjia Li, MD. Department of Ultrasound, Nanfang Hospital, Southern Medical University, Guangzhou 510515, China. Email: wenge67@aliyun.com; Xin Li, PhD. Shenzhen Key Laboratory of Viral Oncology, Center for Clinical Research and Innovation (CCRI), Shenzhen Hospital, Southern Medical University, No. 1333 Xinhu Road, Shenzhen 518100, China. Email: xinli268@gmail.com.

Background: To evaluate the strain ratio (SR) combined with molecular pathological and serum markers for the diagnosis of breast masses.

Methods: SR and 7-point scale elasticity scores were used with real-time tissue elastography and 2-dimensional color-Doppler ultrasound (US) to diagnose breast lesions in 311 hospitalized patients. Immunohistochemical staining and enzyme-linked immunosorbent assays (ELISAs) were used to examine pathological and serum tumor markers and their correlations with SR findings.

Results: SR had a higher diagnostic value compared to the 7-point scale elasticity score, displaying an obvious low-to-high distribution from benign to malignant lesions with an optimal cutoff point at 3.88, which yielded an area under the curve (AUC) of 0.896 with $89.1 \%$ sensitivity, $85.6 \%$ specificity, and positive and negative predictive values of $91.0 \%$ and $82.8 \%$, respectively. The differences of SR values between small $(\leq 1.5 \mathrm{~cm})$, large $(>3 \mathrm{~cm})(\mathrm{P}=0.010)$, and moderate $(>1.5 \mathrm{~cm}$ and $\leq 3 \mathrm{~cm})$ sizes $(\mathrm{P}=0.038)$ in distinguishing benign from malignant breast masses were statistically significant, with SR being most specific and sensitive for diagnosing small lesions. Expression of 3 molecular pathological indicators (p75NTR, p63, and CK5/6), and 5 serum mastocarcinoma markers (uPA, PAI-I, CA27-29, CEA, and CA15-3) showed statistical significance $(\mathrm{P}<0.05)$ in distinguishing between benign and malignant breast lesions. Furthermore, SR combined with CA15-3 and CK5/6 positivity showed $94.2 \%$ sensitivity and $89.2 \%$ specificity as combined markers for triple-negative (TN) breast cancer, whereas SR combined with D2-40 and CK19 were good diagnostic markers for breast cancer lymph node metastasis.

Conclusions: SR, together with a molecular and serological marker, may serve as an additional tool for the diagnosis of small breast cancer tumors.

Keywords: Strain ratio (SR); breast neoplasms; elasticity; diagnostic imaging; elasticity imaging techniques

Submitted Feb 24, 2019. Accepted for publication Feb 12, 2020.

doi: $10.21037 /$ qims.2020.02.14

View this article at: http://dx.doi.org/10.21037/qims.2020.02.14 


\section{Introduction}

Ultrasound elastography (UE) is a non-invasive imaging technique, which facilitates the measurement of tumor elasticity and the early diagnosis of malignant breast tumors (1-3). Although the use of UE has been discredited in diagnosing small nodular breast tumors, previous studies have shown that UE has the advantage of distinguishing and localizing concealed and hard-to-reach small breast lesions missed by routine ultrasound (US) examinations, especially in differentiating malignant from benign tumors $(2,4)$. It is noteworthy that micrometastasis of breast carcinoma in the axillary lymph node is the main cause of treatment failure. Therefore, early and sensitive diagnosis of lymph node micrometastasis would be expected to result in a better prognosis for patients $(5,6)$.

Diagnosis of benign and malignant lesions is generally referred to on a 7-point scale (elasticity score), which is derived from the description by Itoh et al. (7). However, data acquisition and interpretation by different physicians, as well as structural variability within the lesions, could contribute to variable scoring of breast masses $(8,9)$. In contrast, strain ratio (SR) allows semi-quantitative measurement of the lesion stiffness, and, by comparing it with the surrounding tissue, helps to distinguish malignant from benign masses (10). However, there is a discrepancy in the efficiency between the 7-point scoring method and SR for distinguishing malignant from benign masses (10-12).

Our previous study and other reports have shown that the variability in the elasticity of a breast lesion was caused by the stiffness of the mass, which was dependent on the amplification of breast cancer stromal fibroblasts, characterized by the appearance of myofibroblasts (MFS) or carcinoma-associated-fibroblasts $(\mathrm{CAFs})(4,13,14)$. The distribution of MFS in a breast lesion can be identified with a combination of anti-smooth muscle actin ( $\alpha$-SMA) positive and caldesmon negative staining $(4,15,16)$. Other pathological molecules, such as the p75 neurotrophic receptor (p75NTR) (17), tumor suppressor gene p63 and myosin, and keratin 5/6 (CK5/6) (18), have been shown to be expressed in normal but not in tumor tissue. In contrast, expression of the endothelial lymphatic markers D2-40 (19) and keratin 19 (CK19) (20) have been confined to tumor cells that originated from various epithelial cells but were not found in normal lymph node, bone marrow, or peripheral blood, suggesting that these molecules were likely to be useful markers of lymph node micrometastasis.
Furthermore, the American Society of Clinical Oncology (ASCO) performed a literature search on systematic reviews of serum breast tumor markers and confirmed the clinical application value of urokinase plasminogen activator (uPA), plasminogen activator inhibitor-1 (PAI-1), estrogen receptor (ER), progesterone receptor (PR), human epidermal growth factor receptor-2 (HER-2), carcinoembryonic antigen (CEA), CA15-3, and CA27-29 (21) in the diagnosis of small breast lesions. However, to our knowledge, no study has been conducted to explore the association between SR and the molecular pathological and serum markers used in the diagnosis of small breast lesions and lymph node micrometastasis.

Although remarkable improvements have been made in the development of specific molecular markers for breast cancer in recent years, research has not been reported on the combination of markers and UE to facilitate the early diagnosis of breast cancer. In the current prospective study, we first explored the association of SR with UE and tissue and serum molecular markers by integrating imaging, molecular pathological markers (MFS), and serologic data to construct a combined evaluation system to improve the diagnosis accuracy of small breast cancer and micrometastasis of lymph nodes.

\section{Methods}

\section{Study population}

Our prospective study was carried out between 2013 and 2016. A total of 311 consecutive patients scheduled for a core needle biopsy and/or surgical excision were enrolled in our prospective cohort study. Patients who had received any microwave ablation, radiofrequency ablation, etc. or chemical treatment before examinations, such as radiotherapy, immunotherapy and chemotherapy, or had other concurrent malignant tumors, were not included. All of the patients were women [aged 45.0 12.4 years (range, 15-77)]. Of the 311 patients, 208 and 103 were from urban and rural areas, respectively.

All 311 patients underwent routine US, UE at the initial investigation before surgery, and all had histopathological examinations after surgery. Immunohistochemical analyses were performed on 200 breast lesion specimens randomly selected from the 311 patients. There was no statistical deviation after selection. A total of 72 were confirmed to be benign out of 200 lesions using the initial histopathology 
findings as the gold standard. Of the 128 malignant breast lesions, 71 were positive for axillary lymph node metastasis [American Joint Committee on Cancer (AJCC) staging T1N1M0, n=5; T1N2M0, n=2; T2N1M0, n=39; T2N2M0, $\mathrm{n}=15$; T2N3M0, n=8; T2N2M1, n=1; T2N3M1, n=1], and 57 cases were negative. Enzyme-linked immunosorbent assay (ELISA) was carried out on the serum obtained from the 200 patients mentioned above.

\section{Routine US and UE}

B-mode conventional sonography and UE examination for each case were carried out by experienced US physicians simultaneously (Yi Hao, Tao Lv, Xiaoyu Lv, Liang Zhao), and results were only recorded after a consensus of opinion.

Routine US (including 2-dimensional color and spectral Doppler US) was carried out to detect and examine all the solid lesions. Conventional US (including two-dimensional gray-scale US, two-dimensional color Doppler US, and spectral Doppler US) was used to examine all solid lesions, and after comprehensive analyses, masses were classified using the Breast Imaging Reporting and Data System (BI-RADS) into the following: Class I, negative; Class II, benign; Class III, probably benign; Class IV, suspicious malignant; Class V, malignant (22).

UE using a EUP L $54 \mathrm{M}$ scanner $(50 \mathrm{~mm}, 6-13 \mathrm{MHz})$ equipped with elastography software (EUB-8500, Hitachi) was performed in the UE-mode with a pressure bar showing a digital readout [1-7] on the screen, which represented a comprehensive index of external and imposed external force frequencies. We selected readout 3 as the standard in our study. For a large lesion, the border of the solid lesion was included for the elastography assessment. After stable images were obtained, the maximum score of the images was assessed by two well-trained physicians using a 7-point scoring method as follows: (I) even green for the whole breast mass; (II) mosaic mixed with mainly green and minor blue; (III) blue for the core of the lesion and green for peripheral areas; (IV) mosaic mixed with mainly blue and minor green; (V) mainly blue for the lesion (except peripheral areas); (VI) mainly blue for the lesion and peripheral areas; and (VII) extremely blue for the peripheral and main core of the lesion. Scores of $1-4$ and $\geq 5$ were categorized as benign and malignant, respectively.

SR was used to estimate the hardness of the breast lesion and reference values were taken within the fibroglandular tissue at the same depth while the area of the sampling box [region of interest (ROI)] was required to be at least 2 times greater than the mass area, with a distance to the mass of at least $1 \mathrm{~cm}$. The longitudinal dimension of the lesion was measured in B-mode, and the longitudinal elastogram dimension (E) was compared to the B-mode dimension (B) to form an $\mathrm{E} / \mathrm{B}$ ratio. According to the literature, $\mathrm{E} / \mathrm{B}$ ratios $>1$ indicate malignant lesions.

\section{Specimen and puncture methods}

Single hook locating needles under US elasticity imaging guidance were used for preoperative localization. Based on the needle locations, biopsies were collected during surgery and target lesions were taken for paraffin embedding and immunohistochemical staining.

The final diagnosis was established by histopathology after surgical excision or US-guided core needle biopsy. Histopathological diagnoses of the specimens were obtained and served as reference standards. A specialized breast pathologist with 25 years of experience, who was blinded to the results of the US scans, made all the diagnoses.

\section{Immunobistochemical staining and ELISA}

Histopathological staining of all specimens was conducted to confirm either a benign or malignant diagnosis of the breast lesions.

The expression statuses of p75NTR, p63, myosin, and CK5/6 in all 200 specimens, and D2-40 and CK19 in 128 specimens of malignant lesions, were examined by the immunohistochemical 2 staining protocol of Envision (ZSGB-BIO, Beijing, China). Two double-blinded pathologists assessed the results of immunohistochemical staining as the percentage of positively stained cells and the staining intensity (Xinzhi Fang and Yinhua Zhang). Positively stained cells were counted from 100 sampled cells in each high-power field, and the arithmetic mean from 10 representative fields was used to calculate the percentage of positively stained cells, which was scored as $0,1,2$, and 3 for staining cells of $<10 \%, 11-20 \%, 21-50 \%$, and $>50 \%$, respectively. Staining intensity was scored as $0,1,2$, and 3 for no staining, light yellow, light brown, and yellowishbrown, respectively. The accumulated score for each case was expressed as the product of the percentage of positively stained cells and the value of the staining intensity, which was interpreted as negative, positive, and strong positive with accumulated scores of $\leq 2,3-5$, and $\geq 6$, respectively. 
ELISA was used to investigate the expression of various serum mastocarcinoma molecular markers (uPA, PAI-I, PR, ER, HER-2, CEA, CA15-3, and CA27-29) in 200 patients within 3 days before surgery. A total of $5 \mathrm{~mL}$ of nonanticoagulative fasting venous blood was collected in the morning, and the serum was separated by centrifugation. All the serum samples were examined using the specific antibodies, including anti-CK19, D2-40, and p75NTR (Santa Cruz, Santa Cruz, CA, USA), anti-CK5/6 (ZSGBBIO, Beijing, China) and anti-p63 and myosin (Abcam). ELISA kits comprised (CA27-29, CA15-3, antibodiesonline), uPA, PAI-I, PR, ER, HER-2, and CEA (Wuhan UCSN Business Co., Ltd), and all measurements were performed according to the manufacturers' instructions. Standards and samples were pipetted into the wells, and after the proteins were immobilized by their specific antibodies biotinylated secondary antibodies were added after which horse-radish peroxidase (HRP)conjugated streptavidin was added to each well and a tetramethylbenzidine (TMB) substrate solution changed the color from blue to yellow. Each serum sample analysis was repeated 3 times, and the mean value was obtained using an enzymatic-reader (Bio-Rad, Model 680) at $450 \mathrm{~nm}$. A standard curve was established with standard concentrations (Y-axis) and optical density (OD) values (X-axis) using professional software (Curve Expert ver. 1.30). The sample content was determined according to the regression equation obtained from the standard curve and the dilution factor of the sample.

\section{Statistical analysis}

SPSS software (ver. 19.0. IBM Corp., Armonk, NY, USA) was used for all data analyses. A receiver operating characteristic (ROC) curve was plotted using the 7-point scoring assessment and SR against the pathological diagnosis by histopathology, which produced corresponding benign and malignant breast tumor sensitivity, specificity, positive and negative predictive values, false negative and positive rates, and agreement rates. About two-third of the benign and malignant cases were randomly selected by the SPSS software in the benign and malignant groups for immunohistochemical analyses. The measurement data of benign and malignant $\mathrm{SR}$ are presented as the mean $\pm \mathrm{SD}$, and normally distributed data were analyzed using a $t$-test. The pathological diagnosis was used as the gold standard, and an $\chi^{2}$ test was used to compare the best cut-off point obtained from all patients with the diagnostic value of breast lesions in all patients. Variance analysis and a z-test were used to compare the immunohistochemical staining, and ELISA results of the molecular indicators and the area under the curve (AUC) were calculated from the ROC curve. Wilcoxon rank and Spearman's rank correlation tests were used to analyze the correlations between SR and p75NTR, p63, and CK5/6 expression, and to analyze the ordered variables (including uPA, CEA, CA15-3, ER, and $\mathrm{PR}$ ) in serum. The Shapiro-Wilk test was used for normal distribution analysis. $\mathrm{P}$ values $<0.05$ were considered to be statistically significant.

\section{Results}

Of the 311 breast lesions, 118 and 193 were confirmed by histopathological examination to be benign and malignant, respectively, (Figure 1A,B). The most frequent benign and malignant breast mass detected in the present study was breast fibroadenoma $(72 \%, 85 / 118)$ and infiltrating ductal cancer $(72 \%, 139 / 193)$, respectively in the pathological results of all 311 breast lesions (Table S1).

Next, we observed and analyzed the case numbers of benign and malignant breast lesions using a US diagnosis method with a 7 -point scoring system, assessed by 2 independent physicians. Compared to the pathology diagnosis results, we found that the accuracy rate of diagnosis when using an elastographic score $\geq 5$ reached $88.1 \%$ (170/193), and the accuracy rate of benign diagnosis when using an elastographic score $\leq 4$ was $78.8 \%$ (93/118), which suggested a consistency of the 2 evaluation methods in diagnosis efficiency between benign and malignant breast lesions (Table S2, Figure 2).

Furthermore, the diagnosis sensitivity, specificity, as well as the positive and negative predictive values of the 7-point scoring compared with the SR method were $88.1 \%$ vs. $89.1 \%, 78.8 \%$ vs. $85.6 \%, 87.2 \%$ vs. $91.0 \%$, and $80.2 \%$ vs. $82.8 \%$, respectively, whereas the concordance rates between the 7-point scoring and SR data and clinic pathological diagnosis were $84.6 \%$ and $87.8 \%$, respectively (Table S3).

Next, we analyzed the diagnostic rates of the benign and malignant lesions using the SR method and compared the accuracy with the pathological results. The SR of benign lesions was $4.01 \pm 0.65$ (Figure $3 A, B$ ) and that of malignant lesions was $17.62 \pm 1.4$ (Figure $3 C, D$ ), which showed significant differences $(\mathrm{t}=7.29, \mathrm{P}<0.01)$, and suggested that SR might be a useful tool to distinguish benign from 

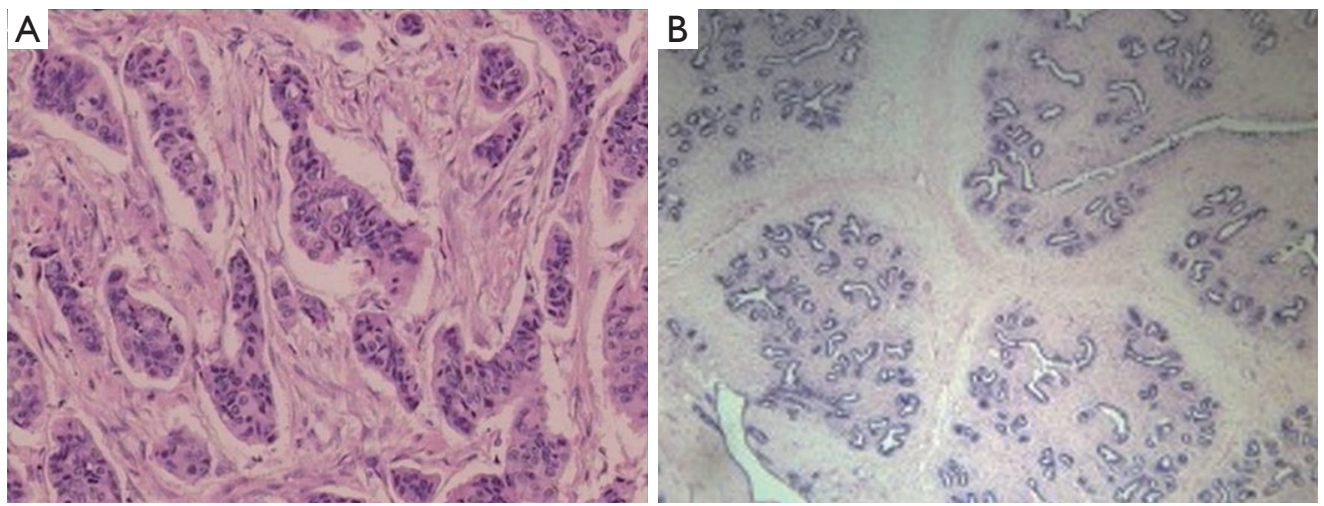

Figure 1 Histopathological examination of benign and malignant breast lesions (A) left breast fibroadenoma, (B) right breast infiltrating ductal cancer (S-P staining, 200× magnification).

malignant breast tumors.

Using the SR data of 311 lesions, a ROC curve was established with 1-specificity as the abscissa and sensitivity as the ordinate. According to the sensitivity and specificity of each possible SR detection in the statistical results, the Youden index (sensitivity + specificity-1) was calculated. The largest Youden index was selected as the cut-off point for diagnosis, and the SR corresponding to this point was determined to be 3.88 with an AUC of 0.896 , indicating that an SR $<3.88$ was a sign of a benign lesion and an SR $\geq 3.88$ was a sign of a malignant lesion. In addition, we found only 21 cases belonging to malignant tissue when using an SR $<3.88$, but 172 cases were malignant and 17 cases benign when using a cut-off value $\geq 3.88$ (Figure 4 ).

To investigate the value of SR in diagnosing various sizes of breast lesions by comparison with routine US BI-RADS assessment categories, the 311 breast lesions were divided into the following 3 groups according to the maximal lesion diameter: small size group $(\leq 1.5 \mathrm{~cm})$, moderate size group ( $>1.5$ and $\leq 3 \mathrm{~cm}$ ), and large size group $(>3 \mathrm{~cm})$. A comparison of the diagnostic methods used between BIRADS scoring and SR is summarized in Table 1. While there was no significant difference in the SR values in the small size group compared with the moderate size group $(\mathrm{P}=0.093)$, the differences between the moderate and large size groups $(\mathrm{P}=0.038)$ and those between the small and large size groups $(\mathrm{P}=0.010)$ were statistically significant. The data showed that the diagnosis sensitivity, specificity, and positive and negative predictive values of SR were $90.7 \%, 89.6 \%$, $92.9 \%$, and $86.7 \%$ for $\leq 1.5$ small size lesions, respectively. A comparison between BI-RADS and SR revealed that SR yielded superior values for small size $(\mathrm{P}=0.008)$ and inferior values for large size $(\mathrm{P}=0.017)$ lesions (Table 1).

Next, we compared tissue and serum biomarkers and SR diagnostic feasibility. The positive expression of p75NTR, p63, myosin, and CK5/6 was observed in benign breast lesions, with a layer of a light-brown heavy line structure on the epithelial cells revealed by immunohistochemical staining (Figure $5 A, B, C, D$ ). In contrast, none or only a faint expression of p75NTR, p63, and CK5/6 was detected in malignant breast lesions (Figure $5 E, F, G, H$ ). Statistical analyses showed that there were significantly different expressions of p75NTR, p63, and CK5/6, but not myosin, in benign and malignant breast masses (Table 2).

Both positive and negative expressions of D2-40 and CK19 were also examined in 128 specimens of infiltrating breast carcinoma (Figure 5I, $7, K, L$ ), and we found moderate to strong positive expression of D2-40 (83.1\%) and CK19 (73.2\%) but negative or a faint D2-40 (16.9\%) and CK19 (26.8\%) expression in 71 metastasis cases. This was somewhat reversed in 57 non-metastasis cases, indicating that both D2-40 and CK19 are markers of axillary lymph node metastasis (Tables $S 4, S 5$ ).

We subsequently examined the expression of several serum molecular markers for mastocarcinoma, including uPA, PAI-I, CEA, CA15-3, CA27-29, ER, PR, and HER-2, using ELISA. According to the ROC curves (Figure 6), the AUCs were $0.615,0.664,0.668,0.702$, and 0.645 for uPA, PAI-I, CEA, CA15-3, and CA27-29, respectively, and the differences of expression in benign and malignant breast lesions were statistically significant $(\mathrm{P}=0.012,0.035,0.026$, 0.000 and 0.027 , respectively), while no differences were 

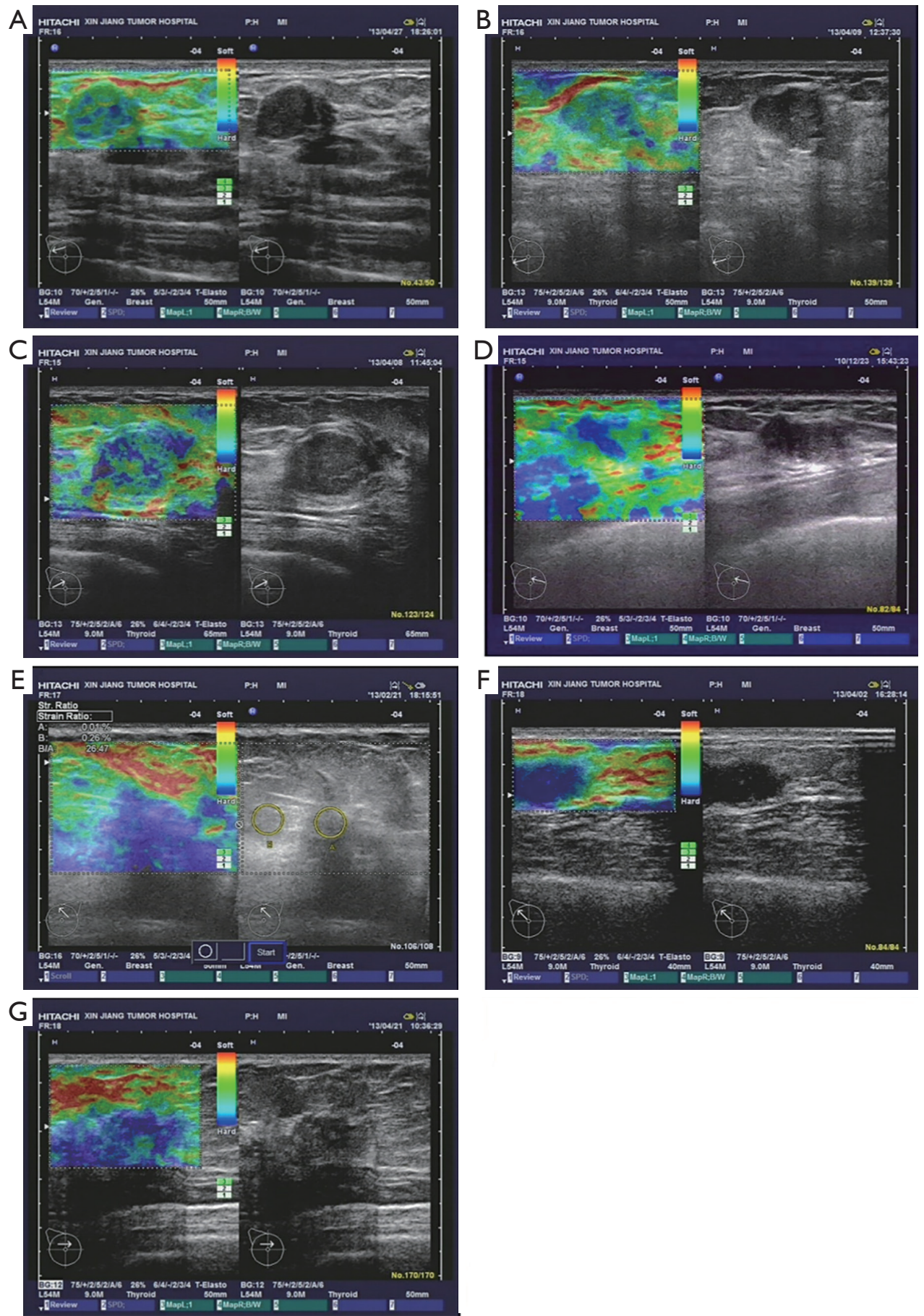

Figure 2 Comparison of pathological diagnosis (right) and 7-point elasticity score assessment (left). (A) Fibroadenoma in the right breast with elasticity score of 1 , showing even green for the solid nodule; (B) fibroadenoma in the right breast with elasticity score of 2 , showing mosaic mixed with mainly green with minor blue for the solid nodule; (C) fibroadenoma in the right breast with elasticity score of 3 , showing green and blue at the core and peripheral region of the solid nodule; (D) fibroadenoma in the right breast with elasticity score of 4, showing mosaic mixed with mainly blue with minor green for the solid nodule; (E) infiltrating ductal carcinoma in the right breast with elasticity score of 5, showing mainly blue for the lesion; (F) infiltrating ductal carcinoma in the left breast with elasticity score of 6 , showing mainly blue for the core and peripheral regions of the lesion; $(G)$ infiltrating ductal carcinoma in the left breast with elasticity score of 7 , showing overwhelming blue for the core and peripheral regions of the lesion mixed with minor green at the mass. 

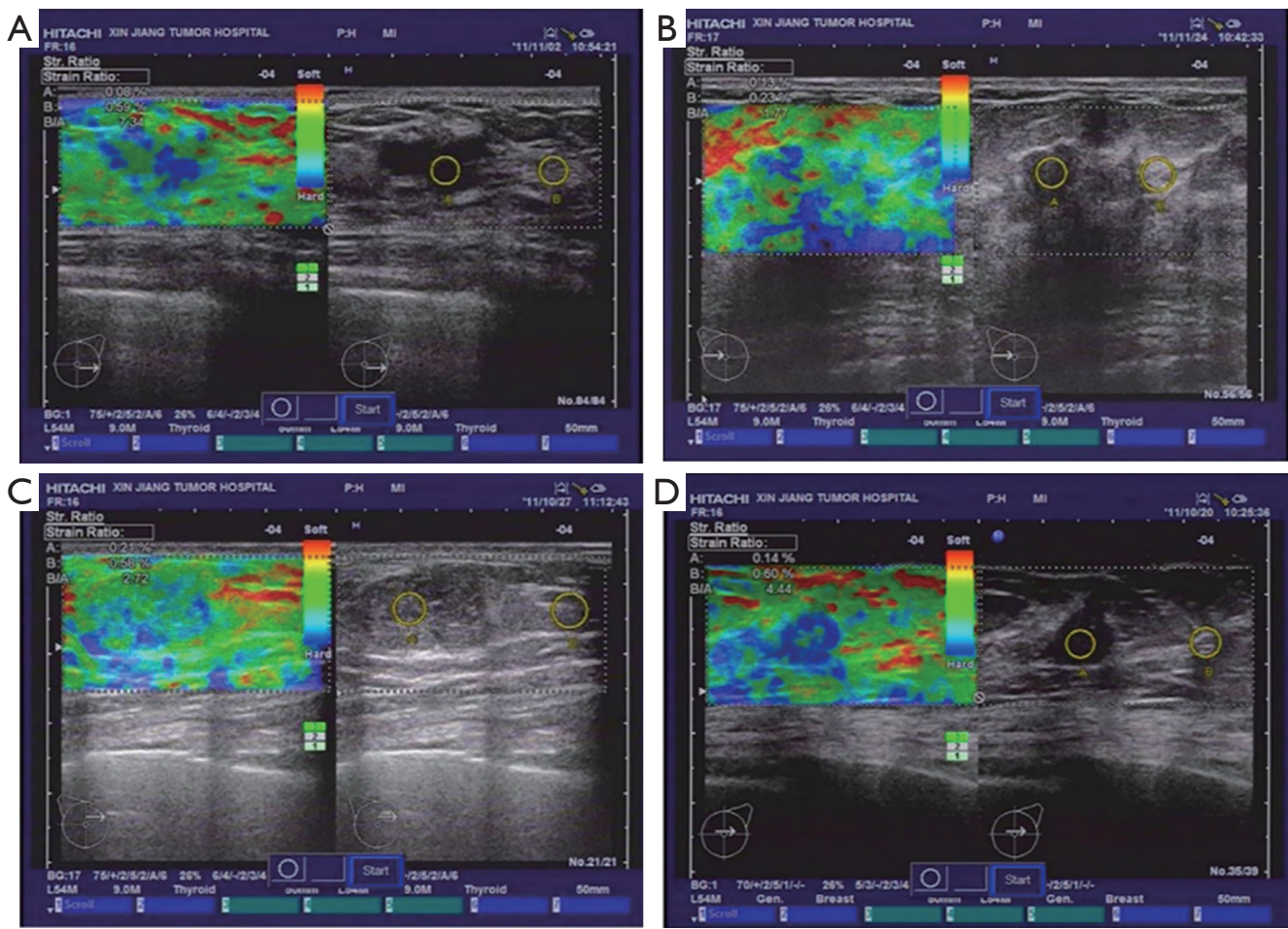

Figure 3 Benign, malignant breast lump SR analysis. (A) Left breast fibroadenoma; (B) right breast fibroadenoma; (C) left breast infiltrating ductal cancer; (D) right breast infiltrating ductal cancer. SR, strain ratio.

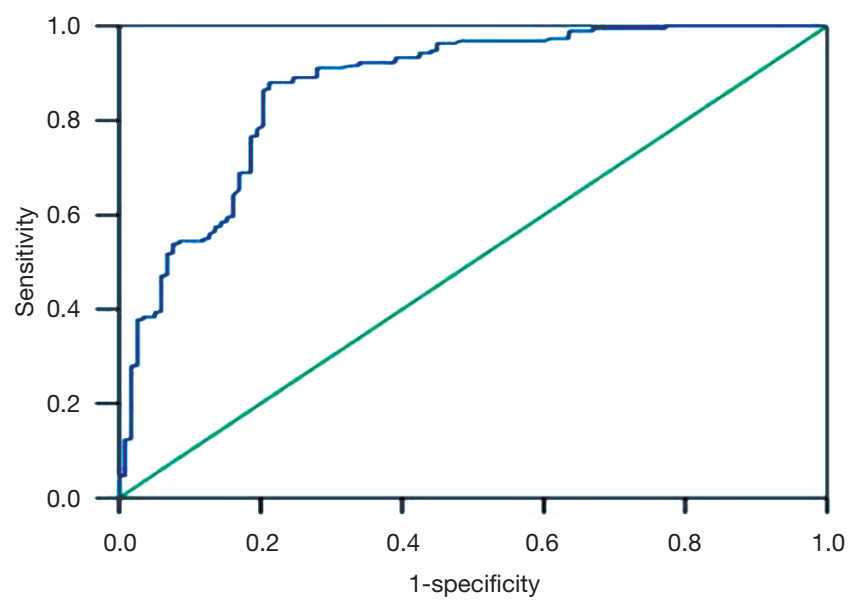

\begin{tabular}{cccc}
\hline \multirow{2}{*}{ SR method } & \multicolumn{2}{c}{ Patho logic diagnosis } & \multirow{2}{*}{ Total } \\
\cline { 2 - 3 } & Malignant & Benign & \\
\hline$\geq 3.88$ & 172 & 17 & 189 \\
$<3.88$ & 21 & 101 & 122 \\
Total & 193 & 118 & 311 \\
\hline
\end{tabular}

Figure 4 ROC for the SR method in 311 breast lesions. ROC, receiver operating characteristic; $\mathrm{SR}$, strain ratio. found for ER, PR, and HER-2, as summarized in Table 3.

Finally, we analyzed the relationship between the SR of breast lesions with pathological and serum mastocarcinoma results. According to the above-mentioned findings, data without statistical significance (myosin and serum PAI-I, CA27-29, and HER-2) or findings only in malignant lesions (D2-40 and CK19) were excluded. Although decreasing relevance was found between SR and p75NTR, and between p63 and CK5/6, statistical significance was noted between SR and CK5/6. In contrast, positive correlations were found between SR and CA15-3, along with a triplenegative $(\mathrm{TN})$ pattern, and the differences in benign and malignant lesions were statistically significant (Table 4).

Table 5 shows that a combination of the marker SR + CA15-3 + CK5/6 yielded a 94.2\% sensitivity and an $89.2 \%$ specificity for the diagnosis of malignant lesions and benign tissues in breast cancer lesions (Table 5).

\section{Discussion}

In UE, SR reflects the extent of tumor hardness and can be 
Table 1 Comparison of diagnostic results between the BI-RADS and SR methods in different sizes of breast lesion groups

\begin{tabular}{|c|c|c|c|c|c|c|c|}
\hline Group & Method & $\begin{array}{c}\text { Sensitivity } \\
(\%)\end{array}$ & $\begin{array}{l}\text { Specificity } \\
(\%)\end{array}$ & $\begin{array}{c}\text { Positive predictive } \\
\text { value (\%) }\end{array}$ & $\begin{array}{c}\text { Negative predictive } \\
\text { value }(\%)\end{array}$ & SR P value & $\begin{array}{c}\text { P value } \\
\text { BI-RADS vs. SR }\end{array}$ \\
\hline $\begin{array}{l}\text { Small size } \\
\text { group }\end{array}$ & BI-RADS & 79.1 & 75.8 & 81.2 & 73.2 & - & 0.008 \\
\hline $\begin{array}{l}\text { Moderate } \\
\text { size group }\end{array}$ & BI-RADS & 83.4 & 77.1 & 85.4 & 79.5 & - & 0.059 \\
\hline $\begin{array}{l}\text { Large size } \\
\text { group }\end{array}$ & SR & 78.6 & 78.6 & 88.0 & 64.7 & $0.010^{\star \star}$ & - \\
\hline
\end{tabular}

${ }^{*}$, Comparison between the small and moderate size group; ${ }^{* *}$, comparison between the small and large size group; ${ }^{* * *}$, comparison between the moderate and large size group. BI-RADS, Breast Imaging Reporting and Data System; SR, strain ratio.

used to distinguish benign and malignant tissue, while the change of tumor hardness causes an alteration in the tumor microenvironment, reflected in the expression of serum tumor markers. The present study explored associations between the strain rate ratio of UE, tissue, and serum molecular markers by integrating imaging, molecular pathology, and serologic data to construct a combined evaluation system to improve the diagnosis accuracy of small breast cancer and micrometastasis of lymph nodes. As a result, a cutoff value of 3.88 was derived for distinguishing between malignant and benign breast masses, which is similar to the cutoff values of 3.5 (23) and 3.77 (24) reported in previous studies.

Compared with traditional B-mode US, UE can effectively differentiate malignant from benign lesions by gathering complete information about tissue elasticity and analyzing the 2-dimensional echocardiogram. The 7 -point scale elasticity score has generally been used for the assessment of elasticity, and a cut off value $\geq 5$ has been suggested to differentiate benign from malignant breast lesions. However, the stability of imaging and subjective interpretation by physicians produces a limitation in accurate diagnosis. SR has been introduced to measure breast lesion hardness and to compare differences between lesions and the surrounding tissue. Consistent with previous studies (25), our investigations have shown that semiquantitative measurement of SR increased the sensitivity, specificity, positive predictive value, and agreement rates for differentiating breast lesions compared with the 7-point scoring method, using pathological diagnosis as the gold standard (summarized in Table S3).

At present, UE is used for the diagnosis of large breast masses, and there has been a discrepancy in its application or the diagnosis of small lesions. However, previous studies (26) have shown great advantages of UE in the diagnosis of small breast lesions with a sensitivity and specificity of $86 \%$ and $100 \%$, respectively. Another study showed that realtime UE had a higher efficiency in distinguishing benign from malignant small lesions of $\leq 1.0 \mathrm{~cm}$ than large lesions $>1.0 \mathrm{~cm}$ (27). In agreement with previous studies, SR was more efficient in diagnosing small and moderate breast lesions than large masses, and the sensitivity and specificity of diagnosis presented a negative correlation with the size of the breast lesions. The reason may be that after malignant lesions gradually grow up, due to the fast growth rate, the internal tissues become loose because of liquefaction and necrotic areas, which results in a hardness decrease in the lesion (28) (Table 1).

The development of molecular pathology is playing a more important role in characterizing breast lesions $(29,30)$, and a variety of early molecular indicators related to the presence of breast cancer will likely be pivotal in guiding clinical practice. Myosin heavy chain (myosin) and CK5/6 are widely expressed in normal interstitial cells and are absent in the interneoplasm. D2-40 and CK19 are expressed in epithelial cells of various tissues such as squamous epithelium, glandular epithelium and mesothelium, and their tumor cells, but not in normal lymph nodes, bone marrow, and peripheral blood, so if they are positive in lymph nodes, lymph node metastases can be readily identified.

Consistent with previous studies, we showed significantly increased expression of p75NTR, p63, and CK5/6 in malignant breast lesions compared to benign masses, 

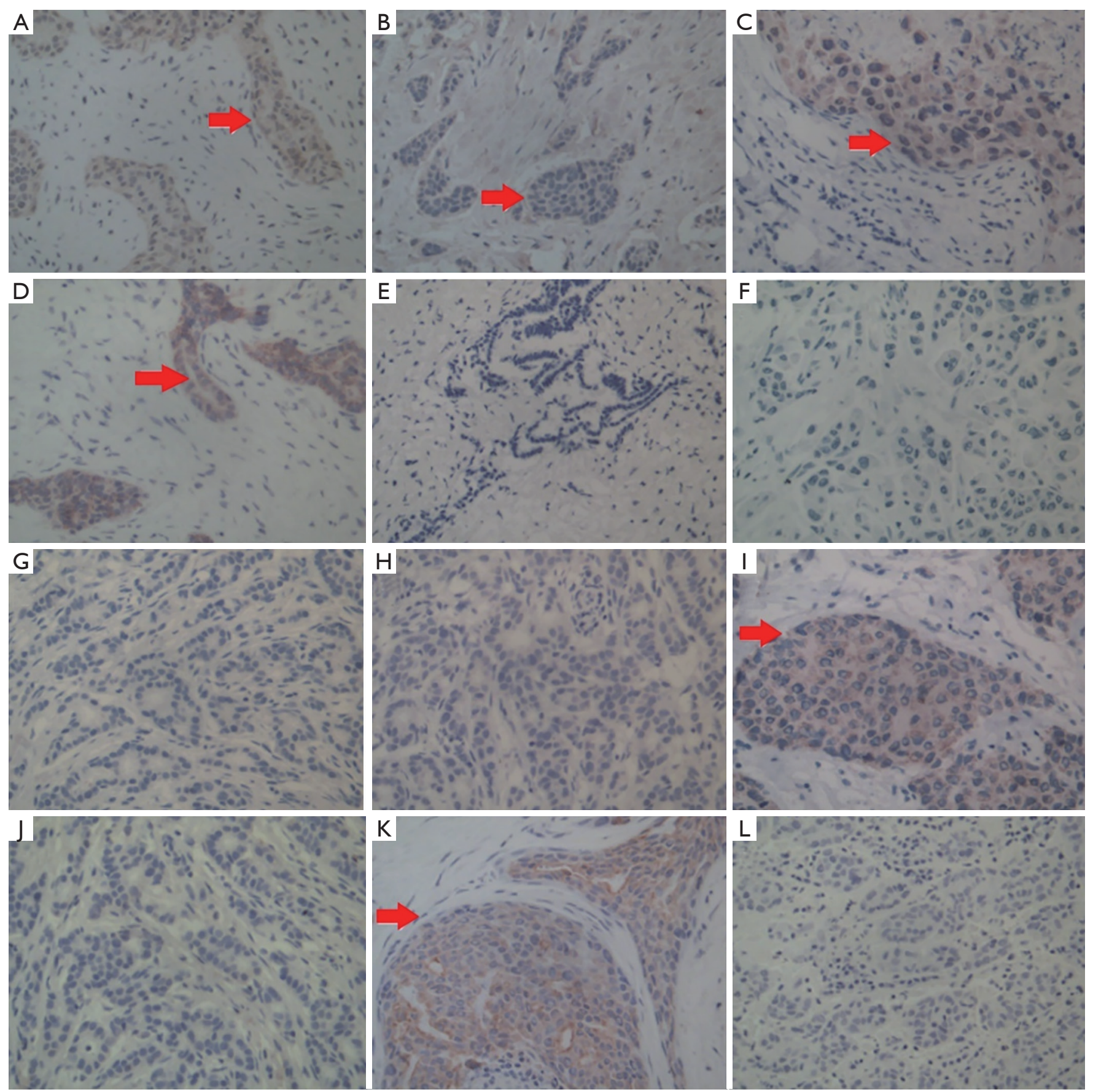

Figure 5 Different molecular pathological indicator expressions in the breast tumor tissues. (A) Positive staining of p75NTR in myoepithelial cells of benign lesions; (B) positive staining of p63 in myoepithelial cells of benign lesions; (C) positive staining of myosin in epithelial cells of benign lesions; (D) positive staining of CK5/6 in ductal epithelial cells of benign lesions; (E) negative staining of p75NTR in breast carcinoma; (F) negative staining of p63 in breast carcinoma; (G) negative staining of p63 in stromal cells of breast infiltrating carcinoma; $(\mathrm{H})$ negative staining of CK5/6 in breast infiltrating ductal carcinoma; (I) strong positive staining of D2-40 in infiltrating breast carcinoma; (J) negative staining of D2-40 in infiltrating breast carcinoma; (K) strong positive staining of CK19 in infiltrating breast carcinoma; (L) negative staining of CK19 in infiltrating breast carcinoma. (S-P staining, 200× magnification). 
Table 2 Comparison of p75NTR, p63, myosin, and CK5/6 expression in 200 breast masses determined by immunohistochemical staining

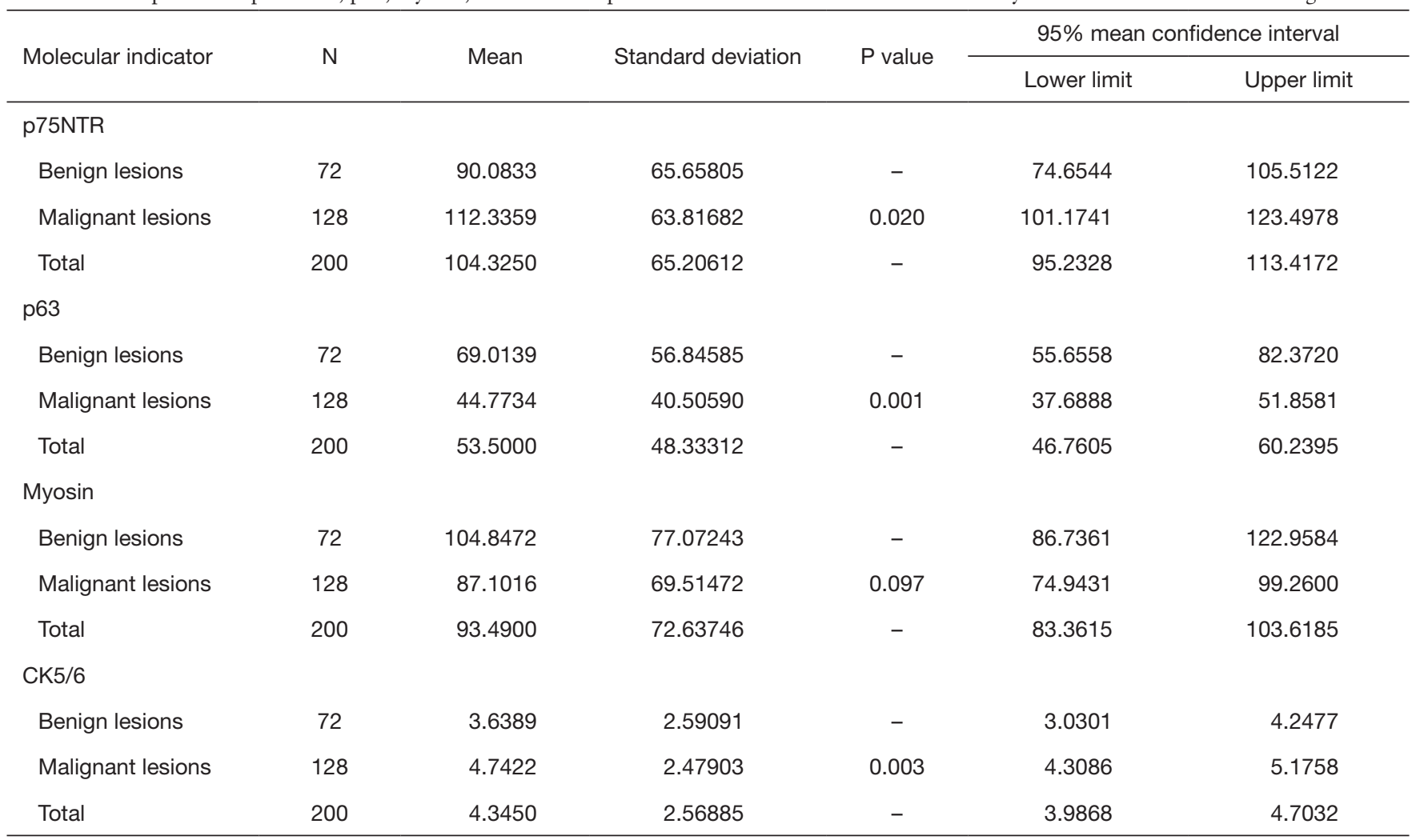

suggesting that these molecules can be used to differentiate benign from malignant breast lesions (31-33). No difference in the expression of myosin was found between malignant and benign lesions, a finding likely caused by the disequilibrium of myosin expression in breast cancer. In the present study, we detected increased and reduced expression of myosin in $\mathrm{TN}$ breast cancer and patients with better prognosis.

Moreover, the expression of D2-40 and CK19 was significantly different between those patients with, and those patients without, axillary lymph node metastasis (Table S4), suggesting the potential of these molecules in evaluating lymph node micrometastasis and patient prognosis.

In recent years, many studies have been performed on the expression of various serum breast tumor markers for non-invasive screening tests for breast cancer (34). By referring to the literature search results of ASCO, in the current study, we used ELISA to examine the expression of various serum molecular mastocarcinoma markers (including uPA, PAI-I, CEA, CA15-3, CA27-29, ER, PR, and HER-2) in breast lesions. Among them, uPA, PAI-I,
CA27-29, CEA, and CA15-3 but not ER, PR, and HER-2, showed statistical significance in distinguishing between benign and malignant breast lesions (Table 3).

Interestingly, significant negative and positive correlations in benign and malignant lesions were confirmed between the SR with CK5/6, and SR with CA15-3 and TN pattern (Table 4), strongly suggesting that the improved diagnostic accuracy of breast lesions could be achieved using the SR combined with corresponding molecular pathological and serum tumor markers (Table 5).

In a previous article, $\mathrm{TN}$ breast cancer tumors were correlated with tumor stiffness, with higher stiffness values than ER+ forms, and the authors concluded that high stiffness values were correlated with aggressive subtypes of breast cancer (35). In our study, TN tumors were also correlated with SR. We thus propose that in TN tumors, cancer-associated fibroblasts might enhance the stiffness via the tumor microenvironment and collagen alterations, as proposed in previous studies $(36,37)$. However, more studies will be required to confirm the correlation between SR and other molecular pathological markers, including $\mathrm{CK} 5 / 6$ and 

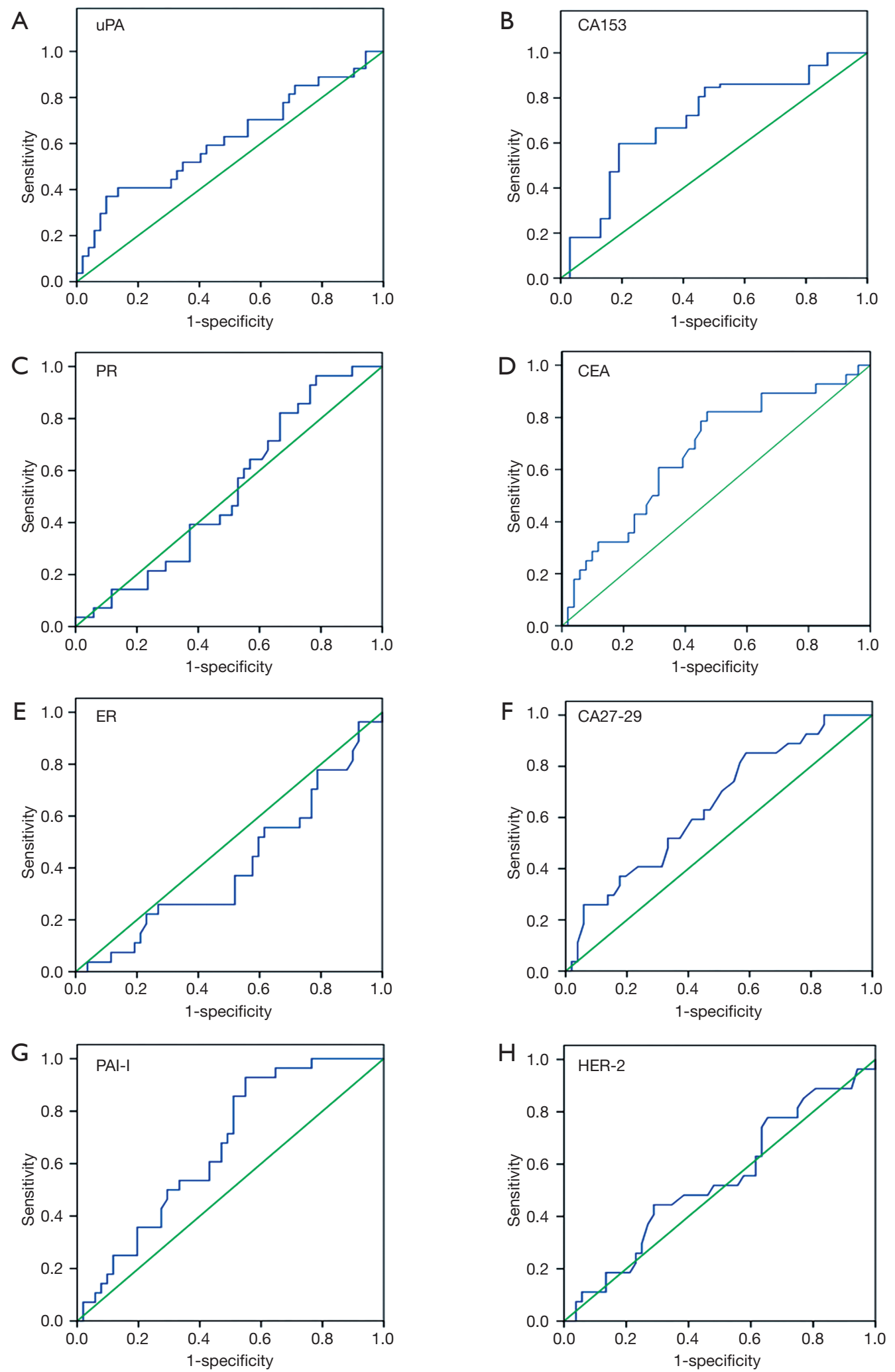

Figure 6 ROC curves of tumor markers. ROC, receiver operating characteristic; uPA, urokinase plasminogen activator; PAI-1, plasminogen activator inhibitor-1; ER, estrogen receptor; PR, progesterone receptor; HER-2, human epidermal growth factor receptor-2; CEA, carcinoembryonic antigen. 
Table 3 Expression of serum molecular markers of mastocarcinoma in benign and malignant breast lesions

\begin{tabular}{|c|c|c|c|c|c|c|c|}
\hline $\begin{array}{l}\text { Tumor } \\
\text { markers }\end{array}$ & Cut-off value & AUC & SE & $95 \% \mathrm{Cl}$ & Sensitivity (\%) & Specificity (\%) & $\begin{array}{c}P \text { value malignant } \\
\text { vs. benign }\end{array}$ \\
\hline uPA & $4.62 \mathrm{ng} / \mathrm{mg}$ & 0.615 & 0.070 & $0.478,0.751$ & 46.911 & 89.625 & 0.012 \\
\hline CEA & $5.2 \mathrm{ng} / \mathrm{mL}$ & 0.668 & 0.064 & $0.543,0.793$ & 43.360 & 98.162 & 0.026 \\
\hline ER & - & 0.408 & 0.068 & $0.276,0.541$ & 34.010 & 94.382 & 0.180 \\
\hline PR & - & 0.521 & 0.066 & $0.392,0.064$ & 31.014 & 90.180 & 0.753 \\
\hline HER-2 & - & 0.536 & 0.060 & $0.400,0.671$ & 30.690 & 92.360 & 0.949 \\
\hline
\end{tabular}

AUC, area under the curve; uPA, urokinase plasminogen activator; PAl-1, plasminogen activator inhibitor-1; ER, estrogen receptor; PR, progesterone receptor; HER-2, human epidermal growth factor receptor-2; CEA, carcinoembryonic antigen.

Table 4 Correlation of SR and molecular pathological indicators (p75NTR, p63, CK5/6) and serum markers (uPA, CEA, CA153, PAI-I, CA27-29, ER, PR, and HER-2)

\begin{tabular}{lcc}
\hline Relative factors & $\mathrm{SR}(\mathrm{r})$ & $\mathrm{P}$ value \\
\hline Pathological indicators & & \\
p75NTR & -0.112 & 0.093 \\
p63 & -0.058 & 0.184 \\
CK5/6 & -0.526 & 0.013 \\
Serum markers & & \\
uPA & 0.131 & 0.100 \\
CEA & 0.058 & 0.184 \\
CA15-3 & 0.621 & 0.038 \\
TN (ER ${ }^{-}$PR $^{-}{ }^{-H E R-2}$ ) & 0.762 & 0.004 \\
\hline
\end{tabular}

$\mathrm{SR}$, strain ratio; TN, triple-negative; uPA, urokinase plasminogen activator; PAl-1, plasminogen activator inhibitor-1; ER, estrogen receptor; PR, progesterone receptor; HER-2, human epidermal growth factor receptor-2; CEA, carcinoembryonic antigen.
CA15-3.

The main limitation of the present study was operator dependence: 1 or 2 radiologists assessed the results of US and UE and decided the final diagnosis. To a certain degree, this is an inevitable drawback of most studies of UE. Fortunately, however, all the radiologists in our study were experienced. Furthermore, SR, as a semi-quantitative method, is more subjective and can avoid inter-operator variability. Another limitation of our research was the small group samples size, which might have limited the accuracy of our conclusions. A multi-center, large sample trial will be conducted shortly to more fully prove our concept.

In conclusion, our results suggest that using the SR with CK5/6 molecular pathological and CA15-3 serum mastocarcinoma markers may improve the accuracy of early diagnosis of small breast cancers, whereas a combination of D2-40 and CK19 markers from axillary lymph nodes might be indicators of early micrometastasis.

Table 5 The evaluation of the diagnostic efficacy of SR index combined with molecular pathologic and tumor serological markers for 311 breast lesions

\begin{tabular}{|c|c|c|c|c|}
\hline Combined diagnostic marker & Sensitivity, \% & Specificity, \% & Positive predictive value, $\%$ & Negative predictive value, $\%$ \\
\hline$S R+C A 15-3$ & 91.5 & 87.2 & 92.3 & 86.6 \\
\hline $\mathrm{SR}+\mathrm{CK} 5 / 6$ & 90.6 & 86.0 & 91.6 & 85.0 \\
\hline $\mathrm{SR}+\left(\mathrm{ER}^{-} \mathrm{PR} \mathrm{R}^{-} \mathrm{HER}-\mathrm{2}^{-}\right)$ & 88.4 & 83.5 & 90.0 & 81.8 \\
\hline $\mathrm{SR}+\mathrm{CA} 15-3+\mathrm{CK} 5 / 6+\left(\mathrm{ER}^{-} \mathrm{PR}^{-} \mathrm{HER}-2^{-}\right)$ & 94.6 & 86.4 & 91.9 & 90.3 \\
\hline
\end{tabular}

SR, strain ratio. 


\section{Acknowledgments}

Funding: This work was supported by the clinical research start-up plan of Southern Medical University (Grant no. LC2016YM018), the Project of Free Exploration from Shenzhen Science and Technology Innovation Commission (Grant no. JCYJ20170307144246792), the Natural Science Foundation of China (Grant no. 81260219), the Natural Science Foundation of China (Grant no. 81671709), the Natural Science Foundation of China (Grant no. 81972423), the Shenzhen Key Laboratory of Viral Oncology (Grant no. ZDSYS201707311140430), the Shenzhen Sanming Medical Project (Grant no.SM201702), and the Science and Technology Planning Project of Guangzhou, Guangdong Province (Grant no. 201804010106).

\section{Footnote}

Conflicts of Interest: The authors have no conflicts of interest to declare.

Ethical Statement: The study was approved by the ethics committee of the Affiliated Tumor Hospital (No. G-201229). All patients provided written informed consent in accordance to the Declaration of Helsinki.

Open Access Statement: This is an Open Access article distributed in accordance with the Creative Commons Attribution-NonCommercial-NoDerivs 4.0 International License (CC BY-NC-ND 4.0), which permits the noncommercial replication and distribution of the article with the strict proviso that no changes or edits are made and the original work is properly cited (including links to both the formal publication through the relevant DOI and the license). See: https://creativecommons.org/licenses/by-nc-nd/4.0/.

\section{References}

1. Lin X, Chang C, Wu C, Chen Q, Peng Y, Luo B, Tang L, Li J, Zheng J, Zhou R, Cui G, Li A, Wang X, Qian L, Zhang J, Wen C, Gay J, Zhang H, Li A, Chen Y. Confirmed value of shear wave elastography for ultrasound characterization of breast masses using a conservative approach in Chinese women: a large-size prospective multicenter trial. Cancer Manag Res 2018;10:4447-58.

2. Shen JH, Luo BM, Ou B, Yang HY. Comparative study of ultrasonic elastography and conventional ultrasonography in differential diagnosis of breast lesions. Chinese Journal of Medical Imaging Technology 2007;23:540-2.
3. Suvannarerg V, Chitchumnong P, Apiwat W, Lertdamrongdej L, Tretipwanit N, Pisarnturakit P, Sitthinamsuwan P, Thiravit S, Muangsomboon K, Korpraphong P. Diagnostic performance of qualitative and quantitative shear wave elastography in differentiating malignant from benign breast masses, and association with the histological prognostic factors. Quant Imaging Med Surg 2019;9:386-98.

4. Hao Y, Guo X, Ma B, Zhu L, Liu L. Relationship between ultrasound elastography and myofibroblast distribution in breast cancer and its clinical significance. Sci Rep 2016;6:19584.

5. Wu X, Lin Q, Chen G, Lu J, Zeng Y, Chen X, Yan J. Sentinel lymph node detection using carbon nanoparticles in patients with early breast cancer. PLoS One 2015;10:e0135714.

6. Yan F, Song Z, Du M, Klibanov AL. Ultrasound molecular imaging for differentiation of benign and malignant tumors in patients. Quant Imaging Med Surg 2018;8:1078-83.

7. Itoh A, Ueno E, Tohno E, Kamma H, Takahashi H, Shiina T, Yamakawa M, Matsumura T. Breast disease: clinical application of US elastography for diagnosis. Radiology 2006;239:341-50.

8. Ricci P, Maggini E, Mancuso E, Lodise P, Cantisani V, Catalano C. Clinical application of breast elastography: state of the art. Eur J Radiol 2014;83:429-37.

9. Yoon JH, Kim MH, Kim EK, Moon HJ, Kwak JY, Kim MJ. Interobserver variability of ultrasound elastography: how it affects the diagnosis of breast lesions. AJR Am J Roentgenol 2011;196:730-6.

10. Zhi H, Xiao XY, Yang HY, Wen YL, Ou B, Luo BM, Liang BL. Semi-quantitating stiffness of breast solid lesions in ultrasonic elastography. Acad Radiol 2008;15:1347-53.

11. Cho N, Moon WK, Kim HY, Chang JM, Park SH, Lyou CY. Sonoelastographic strain index for differentiation of benign and malignant nonpalpable breast masses. J Ultrasound Med 2010;29:1-7.

12. Yerli H, Yilmaz T, Kaskati T, Gulay H. Qualitative and semiquantitative evaluations of solid breast lesions by sonoelastography. J Ultrasound Med 2011;30:179-86.

13. Hua X, Yu L, Huang X, Liao Z, Xian Q. Expression and role of fibroblast activation protein-alpha in microinvasive breast carcinoma. Diagn Pathol 2011;6:111.

14. Tlsty TD. Stromal cells can contribute oncogenic signals. Semin Cancer Biol 2001;11:97-104 .

15. Eyden B, Banerjee SS, Shenjere P, Fisher C. The myofibroblast and its tumours. J Clin Pathol 2009;62:236-49.

16. Yazhou C, Wenlv S, Weidong Z, Licun W. Clinicopathological significance of stromal myofibroblasts 
in invasive ductal carcinoma of the breast. Tumour Biol 2004;25:290-5.

17. Popnikolov NK, Cavone SM, Schultz PM, Garcia FU. Diagnostic utility of p75 neurotrophin receptor (p75NTR) as a marker of breast myoepithelial cells. Mod Pathol 2005;18:1535-41.

18. Moriya T, Kasajima A, Ishida K, Kariya Y, Akahira J, Endoh M, Watanabe M, Sasano H. New trends of immunohistochemistry for making differential diagnosis of breast lesions. Med Mol Morphol 2006;39:8-13.

19. Laser J, Cangiarella J, Singh B, Melamed J, Chiriboga L, Yee H, Darvishian F. Invasive lobular carcinoma of the breast: role of endothelial lymphatic marker D2-40. Ann Clin Lab Sci 2008;38:99-104.

20. Benoy IH, Elst H, Van der Auwera I, Van Laere S, van Dam P, Van Marck E, Scharpé S, Vermeulen PB, Dirix LY. Real-time RT-PCR correlates with immunocytochemistry for the detection of disseminated epithelial cells in bone marrow aspirates of patients with breast cancer. $\mathrm{Br} \mathrm{J}$ Cancer 2004;91:1813-20.

21. Harris L, Fritsche H, Mennel R, Norton L, Ravdin P, Taube S, Somerfield MR, Hayes DF, Bast RC Jr, American Society of Clinical Oncology. American Society of Clinical Oncology 2007 update of recommendations for the use of tumor markers in breast cancer. J Clin Oncol 2007;25:5287-312.

22. D'Orsi C, Sickles E, Morris E. Breast imaging reporting and data system: ACR-BI-RADS-breast imaging atlas, 5th. Reston: American College of Radiology, 2013..

23. Bojanic K, Katavic N, Smolic M, Peric M, Kralik K, Sikora M, Vidačić K, Pacovski M, Stimac D, Ivanac G. Implementation of elastography score and strain ratio in combination with B-mode ultrasound avoids unnecessary biopsies of breast lesions. Ultrasound Med Biol 2017;43:804-16.

24. Khamis MEM, El-deen AMA, Ismail AAA. The diagnostic value of sonoelastographic strain ratio in discriminating malignant from benign solid breast masses. The Egyptian Journal of Radiology and Nuclear Medicine 2017;48:1149-57.

25. Thomas A, Degenhardt F, Farrokh A, Wojcinski S, Slowinski T, Fischer T. Significant differentiation of focal breast lesions: calculation of strain ratio in breast sonoelastography. Acad Radiol 2010;17:558-63.

26. Giuseppetti GM, Martegani A, Di Cioccio B, Baldassarre S. Elastosonography in the diagnosis of the nodular breast lesions: preliminary report. Radiol Med 2005;110:69-76.

27. Barr RG. Real-time ultrasound elasticity of the breast: initial clinical results. Ultrasound Q 2010;26:61-6.

28. Ou B, Luo B, Feng X, Yang H, Zhi H, Wen Y. The value of ultrasonic elastography in differential diagnosing breast small masses. Zhonghua Chaosheng Yingxiangxue Zazhi 2007;16:506-8.

29. Liu H. Application of immunohistochemistry in breast pathology: a review and update. Arch Pathol Lab Med 2014;138:1629-42.

30. Zhao L, Yang X, Khan A, Kandil D. Diagnostic role of immunohistochemistry in the evaluation of breast pathology specimens. Arch Pathol Lab Med 2014;138:16-24.

31. Bhalla A, Manjari M, Kahlon SK, Kumar P, Kalra N. Cytokeratin 5/6 expression in benign and malignant breast lesions. Indian J Pathol Microbiol 2010;53:676-80.

32. Memmi EM, Sanarico AG, Giacobbe A, Peschiaroli A, Frezza V, Cicalese A, Pisati F, Tosoni D, Zhou H, Tonon G, Antonov A, Melino G, Pelicci PG, Bernassola F. p63 Sustains self-renewal of mammary cancer stem cells through regulation of Sonic Hedgehog signaling. Proc Natl Acad Sci U S A 2015;112:3499-504.

33. Verbeke S, Tomellini E, Dhamani F, Meignan S, Adriaenssens E, Xuefen le B. Extracellular cleavage of the p75 neurotrophin receptor is implicated in its pro-survival effect in breast cancer cells. FEBS Lett 2013;587:2591-6.

34. Ouyang Q, Zhang L, Jiang Y, Ni X, Chen S, Ye F, Du Y, Huang L, Ding P, Wang N, Yang C, Huang T, Sun Y, Li S, Xia Y, Hu W, Luo R, Shao Z. The membrane complement regulatory protein CD59 promotes tumor growth and predicts poor prognosis in breast cancer. Int $\mathrm{J}$ Oncol 2016;48:2015-24

35. Chang JM, Park IA, Lee SH, Kim WH, Bae MS, Koo HR, Yi A, Kim SJ, Cho N, Moon WK. Stiffness of tumours measured by shear-wave elastography correlated with subtypes of breast cancer. Eur Radiol 2013;23:2450-8.

36. Alkasalias T, Moyano-Galceran L, Arsenian-Henriksson M, Lehti K. Fibroblasts in the tumor microenvironment: shield or spear? Int J Mol Sci 2018. doi: 10.3390/ ijms 19051532 .

37. Zhou J, Wang XH, Zhao YX, Chen C, Xu XY, Sun Q, Wu HY, Chen M, Sang JF, Su L, Tang XQ, Shi XB, Zhang Y, Yu Q, Yao YZ, Zhang WJ. Cancer-associated fibroblasts correlate with tumor-associated macrophages infiltration and lymphatic metastasis in triple negative breast cancer patients. J Cancer 2018;9:4635-41.

Cite this article as: Hao Y, Ren G, Yang W, Zheng W, Wu Y, Li W, Li X, Li Y, Guo X. Combination diagnosis with elastography strain ratio and molecular markers effectively improves the diagnosis rate of small breast cancer and lymph node metastasis. Quant Imaging Med Surg 2020;10(3):678-691. doi: 10.21037/qims.2020.02.14 


\section{Supplementary}

Table S1 Pathological types of the 311 lesions

\begin{tabular}{lc}
\hline Histopathological diagnosis & Number \\
\hline Malignant lesions & 193 \\
Ductal carcinoma in situ & 12 \\
Infiltrating ductal carcinoma & 139 \\
Infiltrating lobular carcinoma & 23 \\
Mucous carcinoma & 5 \\
Medullary carcinoma & 4 \\
Neuroendocrinoid carcinoma & 1 \\
Medullary carcinoma & 6 \\
Rich in lipid carcinoma & 3 \\
Benign lesions & 118 \\
Mammitis & 7 \\
Mammary adenosis & 16 \\
Papilloma & 10 \\
Fibroadenoma & 85 \\
\hline
\end{tabular}

Table S2 Comparison of elasticity score and pathological diagnosis

\begin{tabular}{lccc}
\hline \multirow{2}{*}{ Elasticity score } & \multicolumn{2}{c}{ Histopathologic diagnosis } & \multirow{2}{*}{ Total } \\
\cline { 2 - 3 } & Malignant & Benign & \\
\hline Malignant & 170 & 25 & 195 \\
Benign & 23 & 93 & 116 \\
Total & 193 & 118 & 311 \\
\hline
\end{tabular}

.

Table S3 Comparison of elasticity scores and SR

\begin{tabular}{|c|c|c|c|c|c|c|}
\hline Object & AUC & Sensitivity, \% & Specificity, \% & $\begin{array}{c}\text { Positive predictive } \\
\text { values, } \%\end{array}$ & $\begin{array}{c}\text { Negative predictive } \\
\text { values, } \%\end{array}$ & $\begin{array}{l}\text { Agreement } \\
\text { rates, \% }\end{array}$ \\
\hline Elasticity score & 0.874 & 88.1 & 78.8 & 87.2 & 80.2 & 84.6 \\
\hline SR & 0.896 & 89.1 & 85.6 & 91.0 & 82.8 & 87.8 \\
\hline
\end{tabular}

$\mathrm{SR}$, strain ratio; AUC, area under the curve.

Table S4 Relationship between D2-40 expression and lymph node metastasis in 128 breast cancer patients

\begin{tabular}{lccc}
\hline D2-40 & \multicolumn{2}{c}{ Axillary lymph node metastasis } & No metastasis \\
\cline { 2 - 3 } & Metastasis & 25 & 34 \\
Moderate-strong positive expression & 59 & 32 & 44 \\
None-faint expression & 12 & 57 & 128 \\
\hline
\end{tabular}

Table S5 Relationship between CK19 expression and lymph node metastasis in 128 breast cancer patients

\begin{tabular}{lccc}
\hline \multirow{2}{*}{ CK19 } & \multicolumn{2}{c}{ Axillary lymph node metastasis } & Notal metastasis \\
\cline { 2 - 4 } & Metastasis & 24 & 76 \\
Moderate-strong positive expression & 52 & 33 & 52 \\
None-faint expression & 19 & 57 & 128 \\
\hline
\end{tabular}

